\title{
On an optimal control strategy in a kinetic social dynamics model
}

\author{
Damián A. Knopoff ${ }^{1,2 *}$, Germán A. Torres ${ }^{3,4}$ \\ ${ }^{1}$ Facultad de Matemática, Astronomía, Física y Computación, Universidad Nacional de Córdoba, Córdoba, \\ Argentina \\ ${ }^{2}$ Centro de Investigación y Estudios de Matemática, CONICET \\ ${ }^{3}$ Facultad de Ciencias Exactas y Naturales y Agrimensura, Universidad Nacional del Nordeste, Corrientes, \\ Argentina \\ ${ }^{4}$ Instituto de Modelado e Innovacion Tecnologica, CONICET \\ *Email address for correspondence: damian.knopoff@unc.edu.ar \\ Communicated by Elena De Angelis \\ Received on 09 29, 2016. Accepted on 04 10, 2018.
}

\begin{abstract}
Kinetic models have so far been used to model wealth distribution in a society. In particular, within the framework of the kinetic theory for active particles, some important models have been developed and proposed. They involve nonlinear interactions among individuals that are modeled according to game theoretical tools by introducing parameters governing the temporal dynamics of the system. In this present paper we propose an approach based on optimal control tools that aims to optimize this evolving dynamics from a social point of view. Namely, we look for time dependent control variables concerning the distribution of wealth that can be managed, for instance, by the government, in order to obtain a given social profile.
\end{abstract}

Keywords: kinetic model, active particles, optimal control, social dynamics

AMS subject classification: 35Q91,90C90,90C11,91A80

\section{Introduction}

This paper deals with an optimal control problem applied to a socio-economic kinetic model for wealth distribution in a society. The objective is to design a suitable strategy that would let an eventual ruler get a desired wealth profile after a certain period of time. The choice of the mathematical model for social dynamics is mainly motivated by the fact that all living systems in general, and social systems in particular, are complex [1], since individuals are able to develop specific heterogeneously distributed strategies, which depend also on those strategies expressed by the other individuals. Moreover, individuals in socio-economic systems are able to learn from their experience, implying that the expression of the strategy evolves in time [2].

The modeling approach is based on the tools of the so called kinetic theory of active particles [3], briefly KTAP, whose essential features can be summarized as follows: the overall system is subdivided into functional subsystems constituted by entities, called active particles, whose individual state is called activity; the state of each functional subsystem is defined by a probability distribution over the activity variable; interactions are, in general, delocalized and nonlinearly additive, and are modeled by stochastic games, where the state of the interacting particles and the output of the interactions can only be known in probability. Finally, the evolution of the probability distribution is obtained by a balance of particles within elementary volumes of the space of the microscopic states, where the dynamics of inflow and outflow of particles is related to interactions at the microscopic scale.

In this way, this approach provides a general mathematical framework that can be specialized into the derivation of a variety of models of complex systems in life sciences. For instance, opinion formation $[4,5]$, immune competition [6], epidemics [7,8], among others, have been properly modeled and described by using this theory. In all of these applications, the heterogeneous behavior of individuals is modeled by 
using theoretical tools of game theory, where the interactions usually involve competitive and altruistic outputs.

More specifically, methods of the kinetic theory have so far been used to model social dynamics. We refer to the recent review paper [9] and references therein for a deep and clear insight into the application of this approach to model social systems. One of the first KTAP-based models was proposed by Bertotti and Delitala [10], and further exploited in [11], where the subpopulations are described by social classes and individuals interact and undergo conservative transitions among these classes. In [12] this model was specialized in order to account for migration from one nation to another. In [13] a focus on the so called black swan [14] in a model linking political and social interactions is introduced. More recently, [2] proposes a model for the onset and development of criminality based on the wealth distribution of a population and [15] links economic development and political competition within a society. Different approaches are compared in [16], while an interesting recent work regarding to the research of a system theory of behavioral social dynamics is presented in [17], where the interested reader is referred to for additional useful references. The book by Pareschi and Toscani is always a valuable query reference in this line of research [18].

In this present paper we consider a spatially homogeneous population divided into functional subsystem characterized by their wealth. Individuals interact with a certain frequency and, as a result of this interaction, they undergo conservative trades that can let them change their social status. Thus, the total population as well as the total wealth remain constant over time. As we will see in the next section, the model can be characterized by a probability of transition from a social class to another and by a threshold that divides altruistic and competitive behaviors, that basically establish the fairness of the wealth distribution in the society. Our main objective is thereby to design an optimal control strategy that would allow an eventual ruler (a controller that can regulate wealth distribution, taxes policies, etc.) to get, within a given period of time, a desired distribution profile. For instance, the ruler may seek for a fairer distribution of the population's resources. With this aim we use some optimal control tools. Namely, we will define a proper functional depending on the state of the system (instantaneous wealth distribution) and on the control variables to be chosen, that has to be minimized through the use of optimization methods in order to obtain a suitable control strategy. Some useful references regarding optimal control of social and economical systems are [19,20]. Some more specific applications can be found, for instance, in $[21,22]$ where suitable developments of the theory are performed.

The paper is organized as follows. Section 2 describes the system under consideration and synthesizes the social model to be used. Section 3 introduces the optimal control problem, by defining an optimization problem in a suitable space. In Section 4 we propose an algorithm and perform a computational analysis in order to obtain suitable controls under different scenarios. Finally, Section 5 is devoted to conclusions and highlights some research perspectives.

\section{The mathematical model}

Let us consider a large population of individuals homogeneously distributed in a certain territory. Individuals are regarded as active particles and they are characterized by a discrete scalar variable $u \in I_{u}=\left\{u_{1}=-1, \ldots, u_{n}=1\right\} \subset[-1,1]$, called the activity, which represents the social state of the individuals, where $u_{1}=-1$ and $u_{n}=1$ are, respectively, the lowest and the highest values of the social state. In general, the study of the model seems to be more interesting when the number $n$ of social classes is odd [10], since in this case a middle class $u_{\frac{n+1}{2}}=0$ can be identified. We recall that the use of a discrete variable is technically convenient since social levels are identified, in practice, only within intervals corresponding to discrete states.

The overall state of the system is described by the generalized distribution functions

$$
f_{i}:[0, T] \rightarrow \mathbb{R}_{\geq 0}, \quad i=1, \ldots, n,
$$

such that $f_{i}(t)$ denotes the number of active particles that, at time $t$, have social state $u_{i}$. The number $T>0$ corresponds to the maximal time of observation and could eventually be $\infty$. We denote by $f(t)$ 
the vector $\left[f_{1}(t), \ldots, f_{n}(t)\right]^{t} \in \mathbb{R}^{n}$. In this way, the total size $N(t)$ of the whole system is given by the sum of all its components:

$$
N(t)=\sum_{i=1}^{n} f_{i}(t)
$$

If the total population is conserved, then $N(t)=N(0)$ for all $t$ and $f$ can be normalized with respect to such a number.

In addition, higher order moments make possible to calculate another macroscopic variables. For instance, the total wealth $W(t)$ is given by the first order moment

$$
W(t)=\sum_{i=1}^{n} u_{i} f_{i}(t)
$$

Moving in this direction, we will use some useful quantities that were introduced in [13]. The number of poor and wealthy active particles at time $t$ are defined by

$$
N^{-}(t)=\sum_{i=1}^{\frac{n-1}{2}} f_{i}(t), \quad N^{+}(t)=\sum_{i=\frac{n+3}{2}}^{n} f_{i}(t)
$$

respectively. In this way, the middle class $u_{\frac{n+1}{2}}=0$ is regarded as economically neutral. If $N$ is normalized, then these quantities satisfy that $0 \leq N^{ \pm} \leq 1$ and $N^{-}+N^{+} \leq 1$ and authors introduced the social gap in the population:

$$
S(t)=N^{-}(t)-N^{+}(t) .
$$

Notice that $S(t) \in[-1,1]$, negative values of $S$ correspond to a wealthy society while positive values of $S$ correspond to a poorer one.

Finally, the variance of the wealth distribution will be also useful in our discussion. It is defined as the sencond order moment with respect to $u$ minus the total wealth, namely

$$
\sigma^{2}(t)=\sum_{i=1}^{n} u_{i}^{2} f_{i}(t)-W(t)^{2} .
$$

Let us now briefly summarize the kinetic social model, referring to $[10,12,13]$ for more details. Interactions involve three types of particles: the test particle, which is representative of the system and whose distribution function is $f_{i}(t)$, candidate particles whose distribution function is $f_{h}(t)$, which interact with field particles whose distribution function is $f_{k}(t)$, which interact with test and candidate particles.

The mathematical structure describes the temporal evolution of the distribution functions $f_{i}$. It is obtained by equating the variation rate of particles in a given functional subsystem $i$, with the difference between the inlet and outlet fluxes from this state. In this way, the balance equation can be summarized as:

$$
\frac{d}{d t} f_{i}(t)=J_{i}[f](t)=G_{i}[f](t)-L_{i}^{j}[f](t), \quad i=1, \ldots, n,
$$

where $J_{i}[f](t)$ is the net flux of particles that fall into the state $u_{i}$, while $G_{i}[f](t)$ and $L_{i}[f](t)$ denote, respectively, the inflow and outflow, at time $t$, into (and out) functional subsystem $i$.

The mathematical framework to describe microscopic interactions between two subsystems can be described by means of two different functions:

- The encounter rate $\eta_{h k}$, that describes the interaction rate between a candidate (or test) $h$-particle and a field $k$-particle. 
- The transition probability density $\mathcal{B}_{h k}^{i}$, that describes the probability density that a candidate $h$-particle falls into the state $u_{i}$ after an interaction with a field $k$-particle. This function satisfies

$$
\sum_{i=1}^{n} \mathcal{B}_{h k}^{i}=1, \quad h, k=1, \ldots, n .
$$

Finally, the equation describing the evolution of the distribution functions can be derived. For $i=$ $1, \ldots, n$ :

$$
\frac{d}{d t} f_{i}(t)=\sum_{h=1}^{n} \sum_{k=1}^{n} \eta_{h k} \mathcal{B}_{h k}^{i} f_{h}(t) f_{k}(t)-f_{i}(t) \sum_{k=1}^{n} \eta_{i k} f_{k}(t) .
$$

Different approaches have been proposed to model the interaction functions. For the case of the interaction rate, we consider the idea from [12], where it was assumed that it depends on the difference between the particles' social states, by considering that the closer the states of the two particles are, the higher is the frequency in which they interact:

$$
\eta_{h k}=\eta_{0}\left(1-\varepsilon\left|u_{h}-u_{k}\right|\right),
$$

where $0 \leq \varepsilon \leq 1 / 2$.

Regarding to the transition probability densities, we take here the cooperative-competitive paradigm. It states that there exists a social threshold $\gamma \in\{1, \ldots, n-1\}$ such that if the distance between the interacting social classes $|h-k|$ is lower or equal that $\gamma$ then individuals undergo a competitive interaction: the richer increases his social state while the poorer decreases it. On the other hand, if $|h-k|>\gamma$, then the opposite (altruistic) effect is observed. In all the cases, it is assumed that transitions are wealth preserving and they occur with probability $\alpha \in[0,1]$. The modeling of this term can be outlined as:

$$
\begin{aligned}
& h=k\left\{\begin{array}{l}
\mathcal{B}_{h h}^{h}=1, \\
\mathcal{B}_{h h}^{i}=0, \quad \forall i \neq h,
\end{array}\right.
\end{aligned}
$$

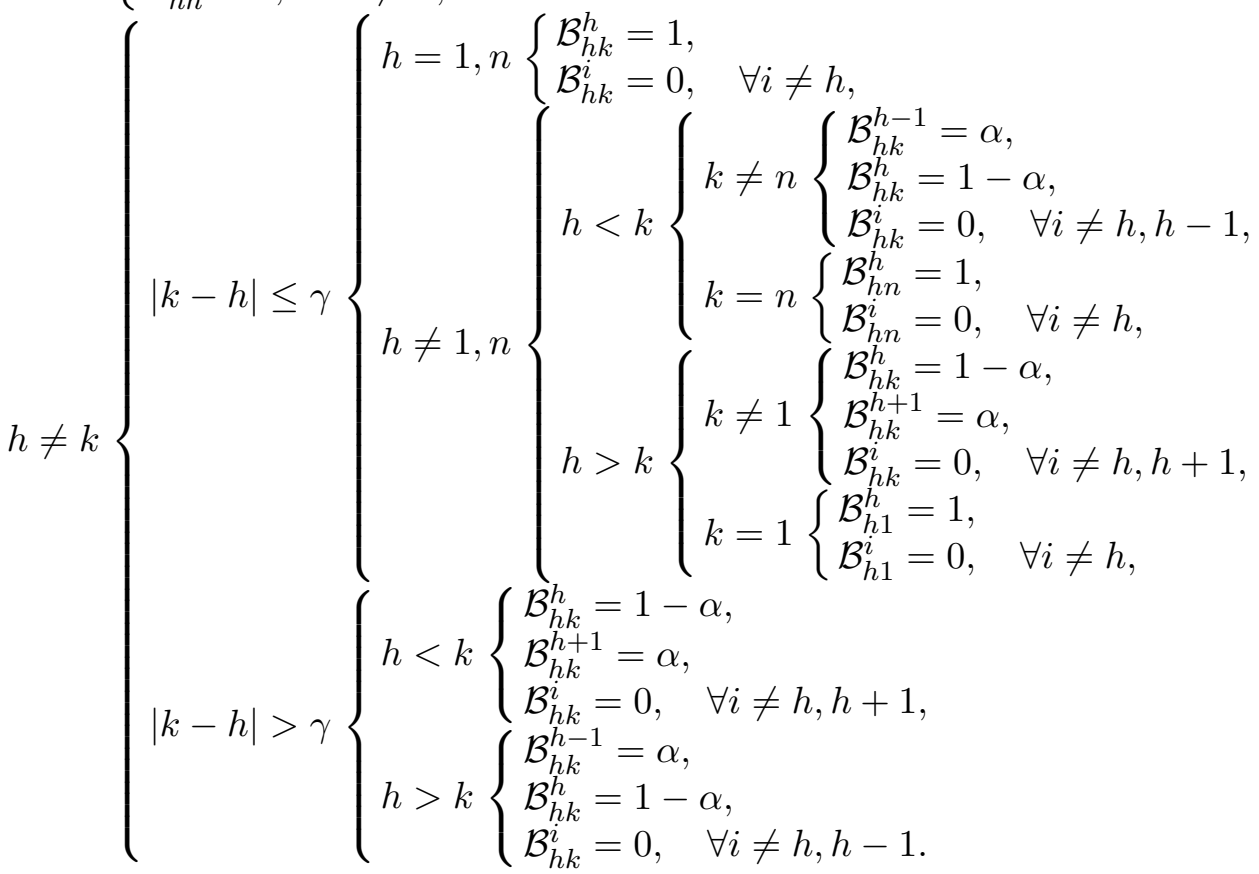

Notice that the model contains two essential parameters that determine the overall dynamics. On the one hand, $0 \leq \alpha \leq 1$ is the probability that, after an interaction, an individual can undergo a transition changing his/her social state. On the other hand, $1 \leq \gamma \leq n-1$ is the critical distance 
that separates competitive from altruistic behaviours. In the first social dynamics models $[10,11] \gamma$ was taken as a constant parameter, while [13] introduced a further development in which $\gamma$ depends on the instantaneous distribution of active particles over the wealth classes.

In this present paper, however, we are interested in the modeling of a situation in which both are viewed as functions of time (in an open loop way) $\alpha(t)$ and $\gamma(t)$ in such a way that they can be controlled (e.g. by a ruler or the government) in order to obtain certain results regarding to the socio-economic distribution in the future. This will be described in detail in the following section.

\section{Formulation of the optimal control problem}

Starting from the previously introduced model, if we emphasize the dependence of $J$ on $\alpha$ and $\gamma$, then eq. (7) can be written as

$$
\begin{aligned}
& \frac{d f}{d t}(t)=J(f(t), a(t)), \quad t \in[0, T], \\
& f(0)=f_{0} .
\end{aligned}
$$

It provides the dynamics of the system, $f:[0, T] \rightarrow \mathbb{R}^{n}$ is called the state of the system and $a$ : $[0, T] \rightarrow A \subset \mathbb{R}^{m}$ is the control function taking values in the admissible region $A$. In our case $m=2$, $a(t)=(\alpha(t), \gamma(t))$ is assumed to be a measurable function and $A=[0,1] \times\{1, \ldots, n-1\}$.

We recall that a pair of functions $(f(\cdot), a(\cdot)):[0, T] \rightarrow \mathbb{R}^{n} \times \mathbb{R}^{m}$ is an admissible pair of the problem if it solves the system given by equation (12).

Let us now put ourselves in the place of the decision maker. His/her aim is to choose an admissible control in an optimal way. In other words, this means that he may select, among all the possible admissible pairs $(f(\cdot), a(\cdot))$ one that minimizes a given cost function, as discussed in the following. The question that he/she would like to answer is:

"I have just risen to power $(t=0)$ and I would like to get, at time $T$, a society with a given social profile. Which policies concerning the distance between social classes and the permeability of the society for wealth exchanges shall I implement in order to obtain my desired social profile?"

In particular, for instance, he/she would like to minimize a given quantity over time and to get, at the end of the process, another quantity as lower as possible. Bearing this in mind, we introduce the following objective function:

$$
P(a(\cdot))=\int_{0}^{T} \varphi(t, f(t), a(t)) d t+\psi(f(T)),
$$

where $\varphi$ can be interpreted as a running cost and $\psi(f(T))$ as a terminal payoff. There are now unique choices for these two functions: for instance, the ruler may pretend to minimize the variance of the wealth distribution $\left(\varphi=\sigma^{2}\right)$ or to maximize the wealthy class $\left(\varphi=-N^{+}\right)$over time, or to keep the social gap at final time as lower as possible $(\psi(f(T))=S(T))$. These and other different choices will be specified in the next section for different case studies.

We are now able to pose the optimal control problem:

$$
\begin{aligned}
\underset{a(\cdot)}{\operatorname{minimize}} & P(a(\cdot)) \\
\text { subject to } & \frac{d f}{d t}(t)=J(f(t), a(t)), \quad t \in[0, T], \\
& f(0)=f_{0}, \\
& f \in\left\{x \in \mathbb{R}^{n}: x_{i} \geq 0, \sum x_{i}=1\right\}, \\
& a(t) \in A, \quad \forall t \in[0, T] .
\end{aligned}
$$

Notice that (14) is a nonlinear constrained optimization problem. It is an optimal control of ODEs problem $[23,24]$, since the state of the system $f$ must solve a system of ordinary differential equations 
given by the social dynamics model (12). In addition, what makes the problem even more challenging is its mixed nature, given that the admissible set $A=[0,1] \times\{1, \ldots, n-1\}$ lets the first variable $(\alpha)$ to be continuous, but the second one $(\gamma)$ is an integer.

Let us remark that problem (14) cannot be tackled with a traditional nonlinear optimization method, since small changes in the variable $\gamma$ do not change the solution of the system of ODEs (12), as can be noted by observing eq. (11). That is why a typical descent or gradient-based method will fail, as it will move in the direction of $\alpha$ only, fixing $\gamma$.

In the next section we propose a numerical method to solve problem (14) and we discuss some selected case studies.

\section{Solving the optimal control problem and numerical results}

\subsection{On the numerical method and design of the algorithm}

In this section we perform some numerical simulations and computational analysis for the optimal control problem presented in Sec. 3. Notice that problem (12) is well-posed and has a unique large-time solution [10,12]. Moreover, notice that if $f_{0}$ has unitary mass, then the condition $f \in$ $\left\{x \in \mathbb{R}^{n}: x_{i} \geq 0, \sum x_{i}=1\right\}$ in (14) is automatically satisfied since the model is conservative. In this case, however, we do not only have to solve the initial value problem but we also need to couple it with the minimization problem. Thus, we must develop an efficient iterative method in order to repeatedly solve (12), evaluate functional (13), adjust the parameter values accordingly, and so on. The method that we use to solve the optimal control problem is summarized in the following algorithm:

\section{Algorithm 4.1. Solving the optimal control problem.}

Step 1: Subdivide the interval $[0, T]$ into $M$ subintervals of the same length, and give an initial piecewise constant guess for the control $a^{(0)}(t)=a^{(0)}\left(t_{k}\right)$, with $t \in\left[t_{k}, t_{k+1}\right], k=0,1, \ldots, M-1$.

Step 2: In the $i$-th step $(i=0,1, \ldots)$, given the initial conditions $f(0)=f_{0}$ and the control $a^{(i)}$, solve the initial value problem (12) to get a solution $f_{k}^{(i)}=f^{(i)}\left(t_{k}\right), k=0, \ldots, M-1$.

Step 3: Evaluate functional (13) to obtain

$$
P\left(a^{(i)}(\cdot)\right)=\int_{0}^{T} \varphi\left(t, f^{(i)}(t), a^{(i)}(t)\right) d t+\psi\left(f^{(i)}\left(t_{M}\right)\right) .
$$

Step 4: Use a convenient method to calculate $a^{(i+1)}\left(t_{k}\right), k=0,1, \ldots, M-1$, such that $P\left(a^{(i+1)}(\cdot)\right) \leq$ $P\left(a^{(i)}(\cdot)\right)$.

Step 5: Return to step 2 and repeat the procedure until the stopping criteria is satisfied.

Some remarks about this algorithm. In step 4 we use a genetic algorithm that takes into account the mixed nature of the constraints; the stopping criteria considered in step 5 is reached when the distance between two consecutive functional evaluations are lower or equal than a given tolerance or when the number of iterations exceed a given allowed maximum.

\subsection{Numerical results}

This subsection is devoted to some simulations that are selected to put in evidence how can a couple of socio-economic parameters let a ruler get a desired social profile in a given population. Therefore, we propose some specific case studies characterized by:

- The initial state of the society: At $t=0$, different initial profiles for wealth distribution are considered, such as poor and rich societies. A random initial distribution is also proposed, since we will see that it is useful to extract some interesting information. In addition, the society is 


\section{Knopoff, G. Torres}

also initially characterized by its values of $\gamma$ and $\alpha$, that give an idea of how fair and permeable interactions are.

- The objective of the controller: The ruler becomes the responsible of the population's administration at $t=0$ and must decide what kind of society he/she glimpses at some future time. Accordingly, he/she will choose the functions $\varphi$ and $\psi$.

The following case studies show, as stated above, different scenarios. In all the cases, we consider nine social classes, namely $n=9$. In addition, the initial threshold that separates competitive from altruistic behaviours is taken large $\gamma(0)=8$ and we take an intermediate value for the probability of experimenting transitions between social classes $\alpha(0)=0.5$. We also take $\eta_{0}=1$ and $\varepsilon=0.5$. Finally, we perform our simulations with $T=100$ and taking $M=100$ time steps.

\subsubsection{Case study 1: Minimizing the variance and the social gap in a fair society}

This first case is quite trivial, but anyways illustrative. Let us consider a society with an initial distribution as shown in Fig. 1a, well-centered around the middle class with a social gap equal to zero. We run our optimization problem taking $\varphi(t, f(t), a(t))=S(t, f(t), a(t))+\sigma^{2}(t, f(t), a(t))$ and $\psi \equiv 0$ in the objective function (13). Namely, the ruler rises to power and would like to minimize, even more, the variance of wealth distribution and the social gap.

First of all, notice that since trades are conservative, the number of wealthy and poor people will coincide in the whole time interval, and consequently $S$ should be equal to zero for all $t$, as observed in Fig. 1f, where oscillations are due to numerical calculations. In addition, the variance effectively decreases as shown in Fig. 1e, with a final distribution shown in Fig. 1b. To get these results, the controller should manage $\alpha$ and $\gamma$ like in Fig. $1 \mathrm{c}$ and $1 \mathrm{~d}$.

Some words about the results. First of all, they are coherent and expectable, but at the same time quite trivial since the society was already fair. Second, we can observe that the implemented policies let the middle class grow. However, the appearance of small clusters in the poorer and wealthier classes could be arguable.

\subsubsection{Case study 2: Minimizing the variance in a poor society}

This case is much more interesting, since we consider a poor society with a very unfair wealth distribution as shown in Fig. 2a. The ruler may take decisions in order to minimize the variance of the distribution, taking $\varphi(t, f(t), a(t))=\sigma^{2}(t, f(t), a(t))$ and $\psi \equiv 0$ in the objective function (13). Applying Algorithm 4.1 to our problem we obtain the final profile shown in Fig. 2b, where we can clearly see that the society, though it is still poor because of the conservative nature of the problen, became fairer. This satisfying result -from the society point of view-is obtained thanks to the application of the policies shown in Fig. 2c for $\gamma(t)$ with a variance evolving as in Fig. 2d.

Notice that, as expected, a fair society is favoured when cooperation is more frequent than competition. In other words, when the critical threshold is kept as lower as possible. Indeed, we can see in Fig. 2c that the ruler may keep $\gamma \equiv 1$ for quite a long time interval: this tells us that a kind of socialist policies should let the government get the desired results.

\subsubsection{Case study 3: Maximizing the wealthy class and minimizing the terminal variance in a poor society}

This case is proposed as a "counterexample" of what should not be done in order to get a fair society. The initial wealth distribution is the same as in case 2 , but the selected functional is obtained by choosing $\varphi(t, f(t), a(t))=-N^{+}(t, f(t), a(t))$ and $\psi(f)=\sigma^{2}$, that is the ruler would like to maximize the number of rich people over the whole time interval and to minimize the variance of the distribution only at final time.

Results are shown in Fig. 3 and we can observe that they are not only alarming but also realistic. Indeed, it is quite normal that a ruler in a poor country tries to favour the few wealthy people (politicians, capitalists, etc.) implementing policies that lead into the situation of Fig. 3b, where the extreme classes 


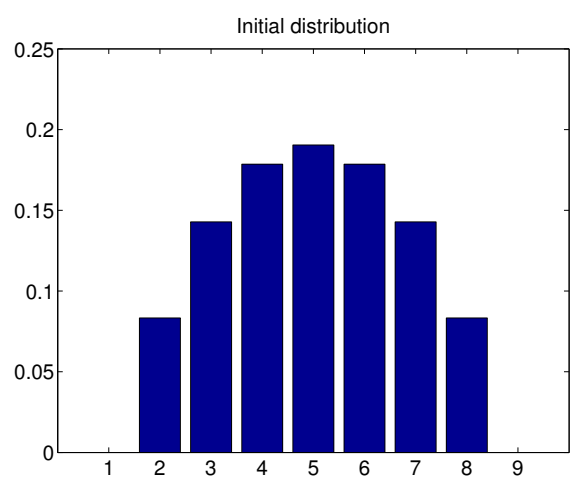

(a)

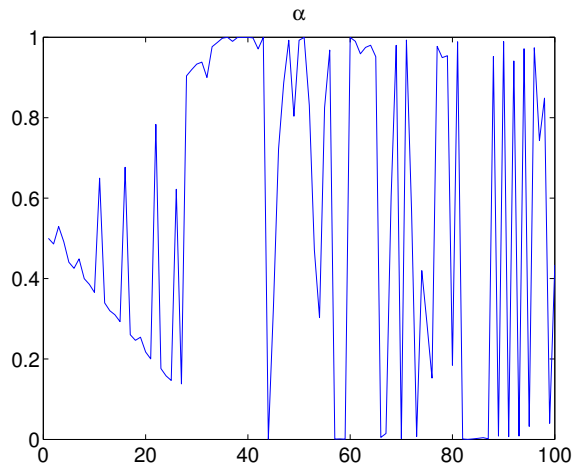

(c)

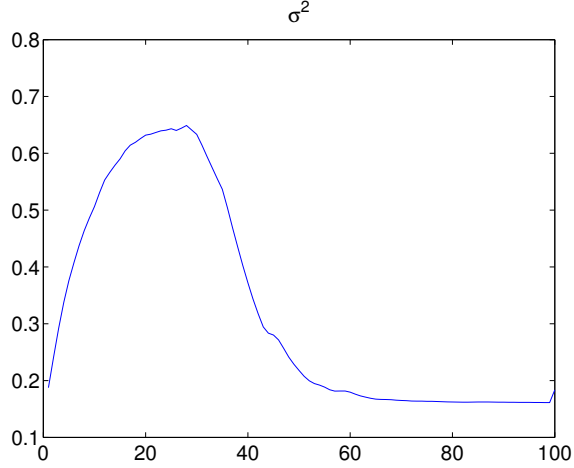

(e)

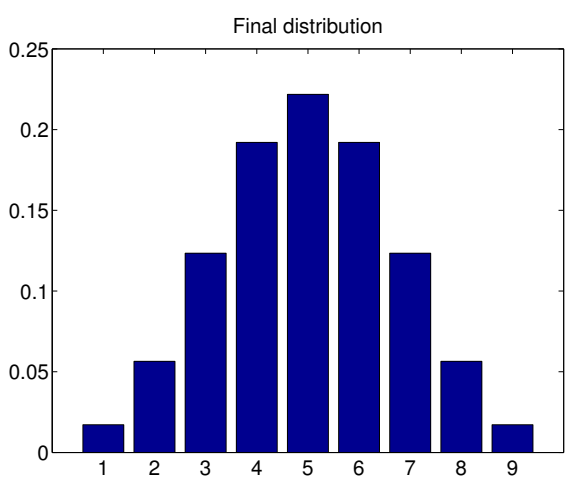

(b)

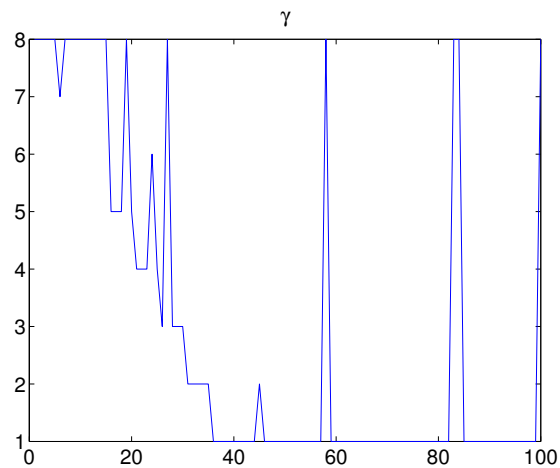

(d)

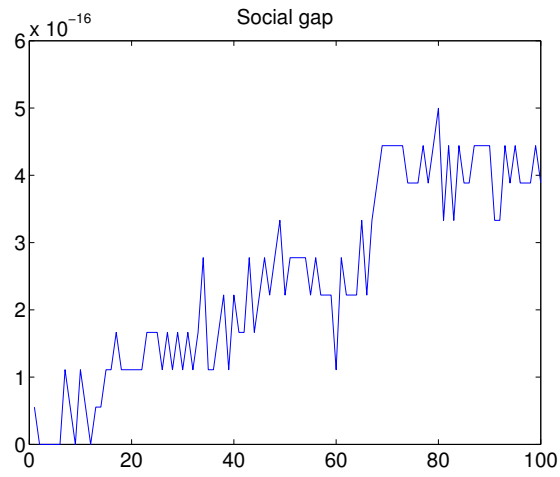

(f)

Figure 1. Case study 1. (a) Initial wealth distribution centered around the middle class with a social gap equal to zero. (b) Wealth distribution at $t=T$. (c) Optimal solution for $\alpha(t)$. (d) Optimal solution for $\gamma(t)$ (integer values are taken only). (e) Variance $\sigma^{2}(t)$ initially increases and then starts to decrease. (f) Social gap $S(t)$ is very close to 0 for all times.

have grown giving as a result an absolutely unfair society. Notice from Fig. 3c that $N^{+}$has effectively increased but at the expense of a huge increase in the variance as seen in Fig. 3d.

\subsubsection{Case study 4: Regularizing a disordered society}

Let us finally consider a toy example in which the society has an initial random profile generated with a normalized uniform distribution, as shown in Fig. 4a. Several experiments have been performed, with different initial random distributions and objective functionals and we found that using $\varphi(t, f(t), a(t))=$ $\sigma^{2}(t, f(t), a(t))-N^{+}(t, f(t), a(t))$ and $\psi \equiv 0$ the ruler is able to obtain the profile shown in Fig. 4b, that represents an almost ideal distribution minimizing the area under the curves in Fig. 4c and Fig 4d. 


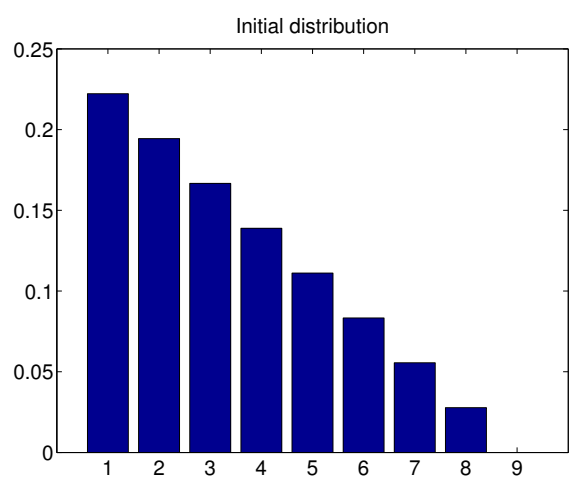

(a)

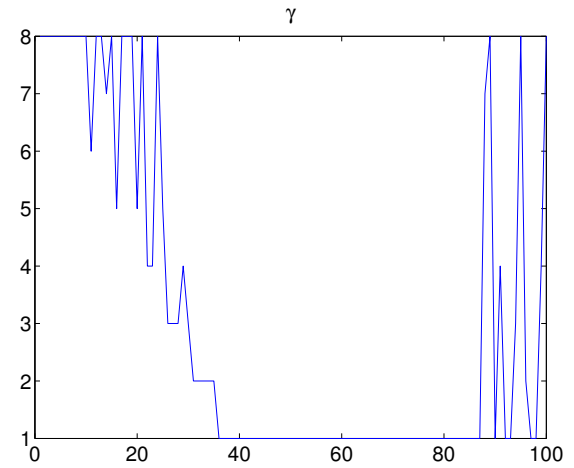

(c)

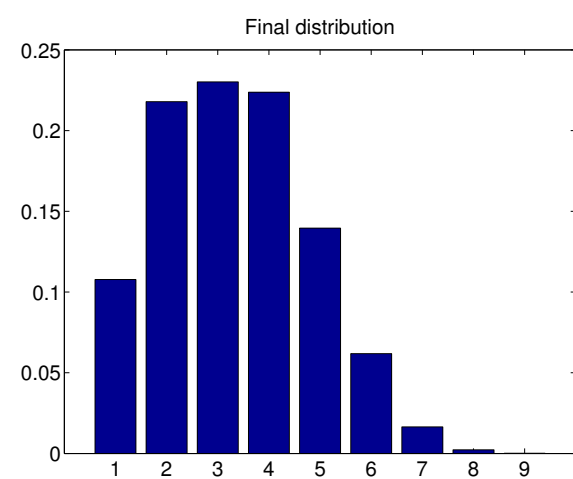

(b)

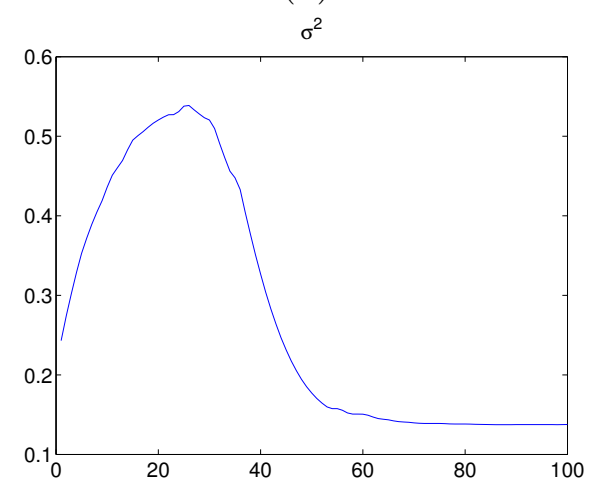

(d)

Figure 2. Case study 2. (a) Initial distribution of a poor society. (b) Wealth distribution at $t=T$. (c) Optimal solution for $\gamma(t)$ (integer values are taken only). (d) Variance $\sigma^{2}(t)$ initially increases and then starts to decrease.

\section{Conclusions and looking forward}

An optimal control problem for a kinetic social dynamics model has been posed in this paper. The main idea is to provide an eventual ruler with certain tools that could let him make strategic decisions in terms of wealth distribution policies, given that -when he rises to power- he has a vision about what kind of society he expects to get in the future. The results obtained in Section 4 show that this method can effectively provide some guidelines and confers utility to the preexisting social models.

The contents of the present paper let us individuate some perspectives for further research activity in the field. First of all, those models concerning social dynamics by means of the kinetic theory [10-12] consider that the total population remains constant over time, by considering a closed conservative system, i.e. a system that does not interact with the outer environment and in which the time interval to be studied is short enough to neglect the development of proliferative or destructive events. However, the assumption of the short-time interval is not such appropriate. In fact, according to the website Breathingeart.net, for instance in China one baby is born every 1.9 seconds and 1 person dies every 3.4 seconds or in Italy a different behavior is observed, with a born every 1.1 minute a death every 50.1 seconds. This suggests that the dynamics of proliferation and destruction should be taken into account in the modeling approach.

Another topic to consider is related to the conservation of wealth as the previous cited models assume that interactions are of such a kind that the total wealth of the system remains constant over time. Nonetheless, the United Nations define the total wealth (of a nation) as a monetary measure which includes the sum of natural (land, forests, fossil fuels, minerals), human (population's education and skills) and physical (machinery, buildings, infrastructure) assets [25]. From this definition it becomes apparent that wealth can be created or destroyed in a society. Indeed, measuring changes in wealth permits to measure the sustainability of development: for instance, the useful report [26] by the World Bank states that, between 1995 and 2005, global wealth increased in per capita terms by 17 percent 


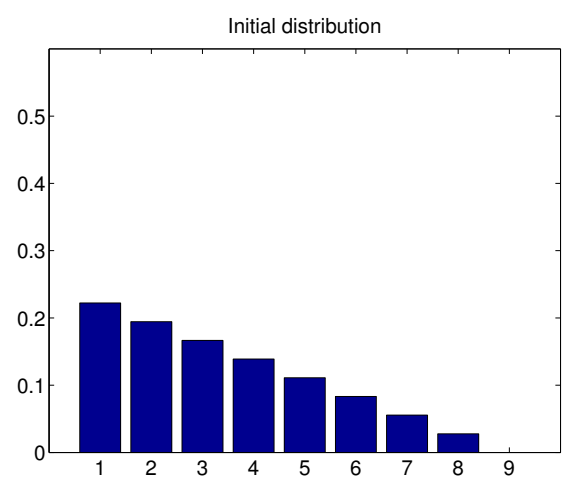

(a)

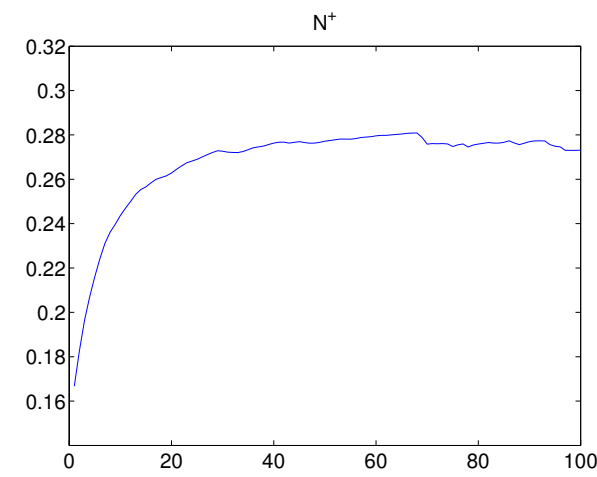

(c)

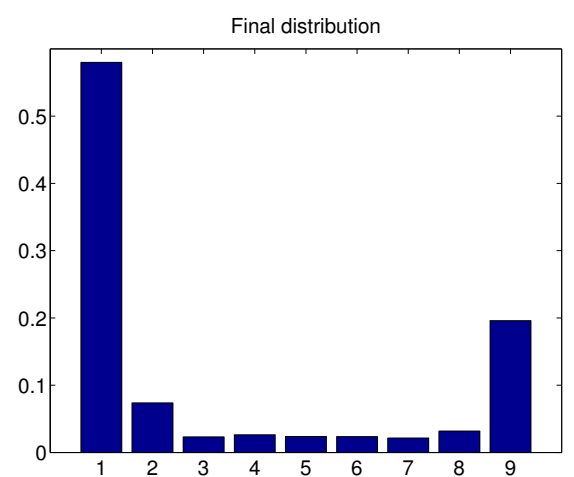

(b)

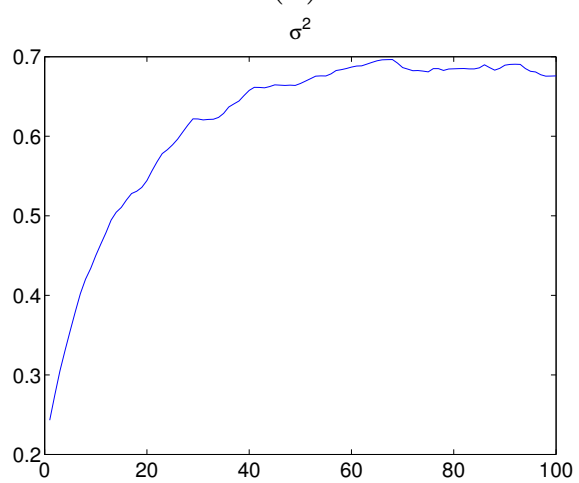

(d)

Figure 3. Case study 3. (a) Initial distribution of a poor society. (b) Wealth distribution at $t=T$. (c) Increasing values of the wealthy class $N^{+}(t)$ (d) Variance $\sigma^{2}(t)$ is increasing, with the society evolving to an even more unequal wealth distribution.

in constant 2005 U.S. dollars. It is also documented that the fastest grow was observed in the lowermiddle-income countries while the majority of the world's wealth (about $82 \%$ ) is hold by the high-income countries in the Organisation for Economic Cooperation and Development (OECD).

In the sake of completeness, the above mentioned aspects shall be combined in order to define wealth per capita. Indeed, if for instance the total wealth of a society increases, but not enough to compensate population growth, then average social welfare will decline. This matter has been slightly taken into account, for instance, in [12] for the modeling of migration phenomena, but it should be further deepen to obtain, not only more interesting models from the mathematical point of view, but also to provide better perspectives to societies that need, as soon as possible, a solution to the problem of wealth distribution.

\section{Acknowledgements}

This work was carried out with the aid of grants from ANPCyT, CONICET and SECyT-UNC.

\section{References}

1. K. D. Bailey, Sociology and the new systems theory: Toward a theoretical synthesis. Suny Press, 1994.

2. N. Bellomo, F. Colasuonno, D. Knopoff, and J. Soler, From a systems theory of sociology to modeling the onset and evolution of criminality, Networks 83 Heterogeneous Media, vol. 10, no. 3, 2015.

3. N. Bellomo, D. Knopoff, and J. Soler, On the difficult interplay between life, "complexity", and mathematical sciences, Mathematical Models and Methods in Applied Sciences, vol. 23, no. 10, pp. 1861-1913, 2013.

4. M. Dolfin and M. Lachowicz, Modeling opinion dynamics: how the network enhances consensus, Networks \&3 Heterogeneous Media, vol. 10, no. 4, pp. 421-441, 2015.

5. D. Knopoff, On a mathematical theory of complex systems on networks with application to opinion 


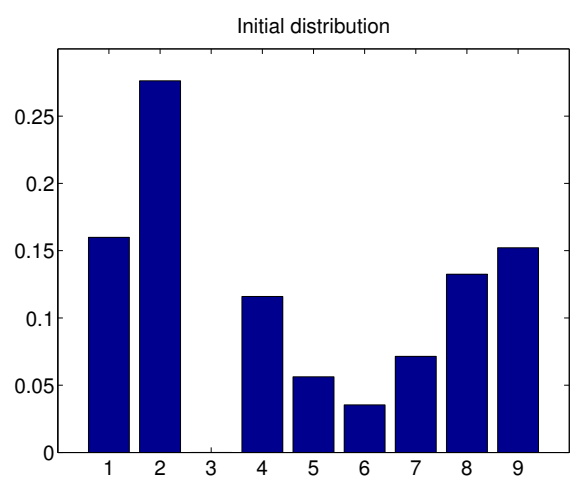

(a)

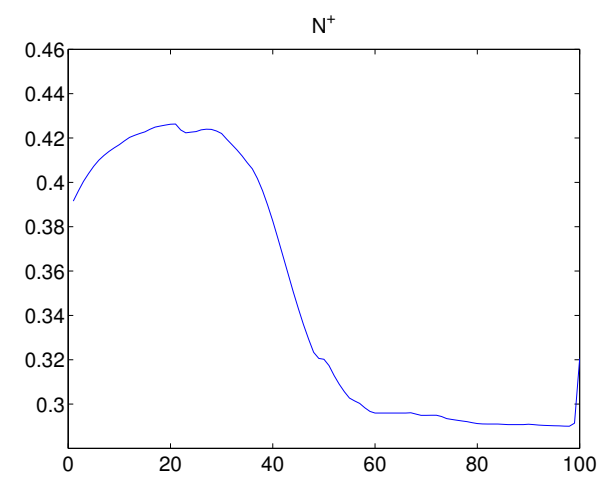

(c)

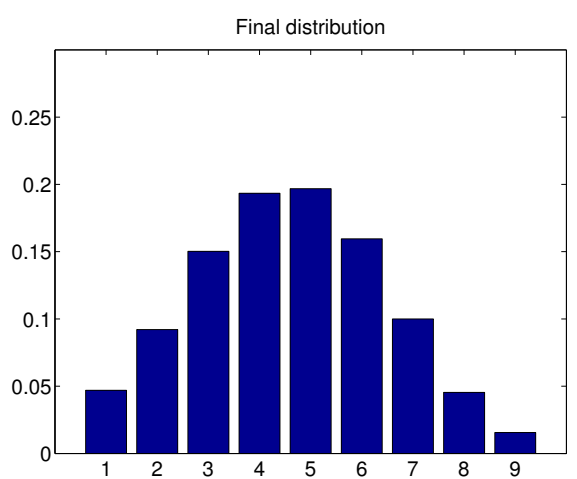

(b)

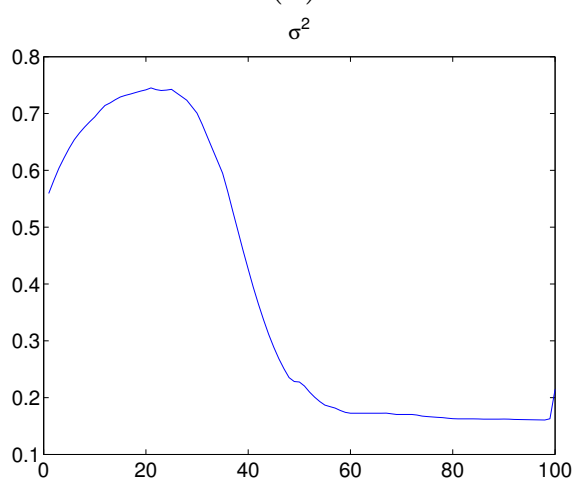

(d)

Figure 4. Case study 4. (a) Randomly generated initial distribution of wealth. (b) Wealth distribution at $t=T$. (c) Wealthy class population $N^{+}(t)$ (d) Variance $\sigma^{2}(t)$.

formation, Mathematical Models and Methods in Applied Sciences, vol. 24, no. 2, pp. 405-426, 2014.

6. A. Bellouquid, E. De Angelis, and D. Knopoff, From the modeling of the immune hallmarks of cancer to a black swan in Biology, Mathematical Models and Methods in Applied Sciences, vol. 23, no. 5, pp. 949-978, 2013.

7. D. A. Knopoff and J. M. Sánchez Sansó, A kinetic model for horizontal transfer and bacterial antibiotic resistance, International Journal of Biomathematics, vol. 10, no. 04, p. 1750051, 2017.

8. M. Delitala, P. Pucci, and M. Salvatori, From methods of the mathematical kinetic theory for active particles to modeling virus mutations, Mathematical Models and Methods in Applied Sciences, vol. 21, no. supp01, pp. 843-870, 2011.

9. M. Dolfin, L. Leonida, and N. Outada, Modeling human behavior in economics and social science, Physics of Life Reviews, 2017.

10. M. L. Bertotti and M. Delitala, From discrete kinetic and stochastic game theory to modelling complex systems in applied sciences, Mathematical Models and Methods in Applied Sciences, vol. 14, no. 7, pp. 1061-1084, 2004.

11. M. L. Bertotti and M. Delitala, Conservation laws and asymptotic behavior of a model of social dynamics, Nonlinear Analysis: Real World Applications, vol. 9, no. 1, pp. 183-196, 2008.

12. D. Knopoff, On the modeling of migration phenomena on small networks, Mathematical Models and Methods in Applied Sciences, vol. 23, no. 3, pp. 541-563, 2013.

13. N. Bellomo, M. A. Herrero, and A. Tosin, On the dynamics of social conflicts: looking for the black swan, Kinetic and related models, vol. 6, no. 3, pp. 459-479, 2013.

14. N. N. Taleb, The black swan: The impact of the highly improbable. Random house, 2007.

15. M. Dolfin, D. Knopoff, L. Leonida, and D. M. A. Patti, Escaping the trap of "blocking": a kinetic model linking economic development and political competition, Kinetic and Related Models, vol. in press, 2016. 
16. B. Düring, D. Matthes, and G. Toscani, Kinetic equations modelling wealth redistribution: a comparison of approaches, Physical Review E, vol. 78, no. 5, p. 056103, 2008.

17. G. Ajmone Marsan, N. Bellomo, and L. Gibelli, Stochastic evolutionary differential games toward a systems theory of behavioral social dynamics, Mathematical Models and Methods in Applied Sciences, vol. 26, no. 6, pp. 1051-1093, 2016.

18. L. Pareschi and G. Toscani, Interacting multiagent systems: kinetic equations and Monte Carlo methods. OUP Oxford, 2013.

19. D. Cass and K. Shell, The Hamiltonian approach to dynamic economics. Academic Press, 2014.

20. W. Stadler, Multicriteria Optimization in Engineering and in the Sciences, vol. 37. Springer Science \& Business Media, 2013.

21. G. Albi, M. Herty, and L. Pareschi, Kinetic description of optimal control problems and applications to opinion consensus, Communications in Mathematical Sciences, vol. 13, no. 6, pp. 1407-1429, 2015.

22. A. Barrea and M. E. Hernández, Optimal control of a delayed breast cancer stem cells nonlinear model, Optimal Control Applications and Methods, vol. 37, no. 2, pp. 248-258, 2016.

23. M. Gerdts, Optimal control of ODEs and DAEs. Walter de Gruyter, 2012.

24. S. Lenhart and J. T. Workman, Optimal control applied to biological models. Crc Press, 2007.

25. UNU-IHDP., Inclusive wealth report 2012: measuring progress toward sustainability. Cambridge University Press, 2012.

26. M. Jarvis, G.-M. Lange, K. Hamilton, D. Desai, B. Fraumeni, B. Edens, S. Ferreira, H. Li, L. Chakraborti, W. Kingsmill, et al., The changing wealth of nations: measuring sustainable development in the new millennium. 2011. 